OPEN ACCESS

Edited by:

Brian Godman,

Karolinska Institutet (KI), Sweden

Reviewed by:

Antony Paul Martin,

University of Liverpool,

United Kingdom

Daniel R. Faleiros,

Universidade de Brasilia, Brazil

*Correspondence:

David Bin-Chia Wu

david.wu@monash.edu

${ }^{\dagger}$ These authors have contributed equally to this work

Specialty section:

This article was submitted to Pharmaceutical Medicine and Outcomes Research,

a section of the journal

Frontiers in Pharmacology

Received: 18 July 2018 Accepted: 06 September 2018

Published: 16 October 2018

Citation:

Wu DB-C, Yee CH, Ng C-F, Lee SWH,

Chaiyakunapruk N, Chang Y-S and Lee KKC (2018) Economic Evaluation

of Combination Therapy Versus Monotherapy for Treatment of Benign Prostatic Hyperplasia in Hong Kong.

Front. Pharmacol. 9:1078. doi: 10.3389/fphar.2018.01078

\section{Economic Evaluation of Combination Therapy Versus Monotherapy for Treatment of Benign Prostatic Hyperplasia in Hong Kong}

\author{
David Bin-Chia Wu $\mathbf{W}^{1,2 * t}$, Chi Hang Yee ${ }^{3 \dagger}$, Chi-Fai $\mathrm{Ng}^{3}$, Shaun Wen Huey Lee ${ }^{1,2}$, \\ Nathorn Chaiyakunapruk ${ }^{1,2,4,5}$, Yu-Shan Chang ${ }^{6}$ and Kenneth Kwing Chin Lee ${ }^{1}$ \\ ${ }^{1}$ School of Pharmacy, Monash University Malaysia, Bandar Sunway, Malaysia, ${ }^{2}$ Asian Centre for Evidence Synthesis \\ in Population, Implementation and Clinical Outcomes, Health and Well-being Cluster, Global Asia in the 21st Century (GA21) \\ Platform, Monash University Malaysia, Bandar Sunway, Malaysia, ${ }^{3}$ Division of Urology, Department of Surgery, The Chinese \\ University of Hong Kong, Shatin, Hong Kong, ${ }^{4}$ School of Pharmacy, University of Wisconsin, Madison, WI, United States, \\ ${ }^{5}$ Department of Pharmacy Practice, Faculty of Pharmaceutical Sciences, Center of Pharmaceutical Outcomes Research, \\ Naresuan University, Phitsanulok, Thailand, ${ }^{6}$ College of Commerce, Tamkang University, New Taipei City, Taiwan
}

Background: Lower urinary tract symptoms (LUTS) suggestive of benign prostatic hyperplasia (BPH) is a common condition affecting men. Studies have shown that the prevalence of LUTS/BPH increases with age, which will cause considerable economic burden to the healthcare system and society. The aim of the present study was to evaluate the long term cost effectiveness of dutasteride and tamsulosin therapy compared to tamsulosin alone in men with $\mathrm{BPH}$ in Hong Kong.

Methods: A Markov decision model was constructed to estimate the economic impact from a healthcare payers' perspective, which only included direct costs. Analyses were conducted for a 4-year time frame.

Results: When compared to tamsulosin alone, combination therapy was more expensive but also more effective in preventing complications and reduced the need for surgery. Over life-time projection suggest that combination therapy will be cost-effective if the willingness-to pay threshold of USD 20,000.

Conclusion: Findings of this study found that combination therapy of tamsulosin and dutasteride was more cost-effective compared to tamsulosin alone across a wide range of scenario.

Keywords: benign prostatitc hypertrophy, cost effectiveness, dutasteride, economic, combination therapy

\section{INTRODUCTION}

Lower urinary tract symptom (LUTS) suggestive of benign prostatic hyperplasia (BPH) is a common condition among middle-aged to elderly men. It is characterized by a cluster of chronic urinary symptoms in the bladder, prostate, and a major cause of benign prostatic hyperplasia. Population based studies suggested that nearly one in every four men aged 50 and above is affected by LUTS (Lee et al., 2017). Similarly, a recent study found that $69.3 \%$ of community-dwelling men experienced moderate-to-severe symptoms on International Prostate Symptom Score (IPSS) in Hong Kong (Yee et al., 2014). Without proper treatment, LUTS can present with complications, 
such as acute urinary retention (AUR), urinary tract infections, or sometimes obstructive uropathy (Lee et al., 2007;McVary et al., 2011). In the current era of an increased life expectancy and the aging of baby boomer generation, male LUTS would become an issue of increasing socioeconomic and medical importance (Lee et al., 2008; Kaplan et al., 2015; Lee et al., 2017).

The main pharmacological agents for the management of LUTS are alpha-blockers and 5-alpha-reductase inhibitors (5ARIs). Currently, international guidelines (McVary et al., 2011; Oelke et al., 2013) recommend the use of alphablockers for symptomatic relief in LUTS patients who do not have a markedly enlarged prostate, while highlighting that these agents do not alter the natural progression of the disease. On the other hand, 5ARIs, which can be administered as monotherapy or in combination with alphablockers, are recommended for symptomatic men with an enlarged prostate and are associated with decreased risk of urinary retention and related surgery. One of the major trials to support such recommendation is the Combination of Avodart ${ }^{\mathrm{TM}}$ (dutasteride) and tamsulosin (CombAT) trial (Roehrborn et al., 2010, 2011). In this randomizedmulticenter, double-blind, parallel-group study of 50 years or older men with a clinical diagnosis of moderate to severe $\mathrm{BPH}$, a single-dose tamsulosin/dutasteride combination therapy was compared with tamsulosin and dutasteride monotherapies. It was found that patients with a prostate volume $\geq 40 \mathrm{ml}$ had a lower risk of disease progression, AUR, and $\mathrm{BPH}$-related surgery in the groups receiving dutasteride or combination therapies than in the group receiving tamsulosin monotherapy.

In terms of economic burden, BPH/LUTS is associated with high personal and societal costs, which are evident in direct medical costs and indirect losses in daily functioning, and through its negative impact on quality of life (QoL) for both patients and partners (Lee et al., 2008; Speakman et al., 2015). Treatment and interventions for BPH/LUTS are essential, which aim at providing symptom relief as well as addressing the root cause, while limiting the occurrence of adverse events. As the prevalence of BPH/LUTS increases with age, the burden on the healthcare system and society may increase due to the aging population (Rosen et al., 2003; Lee et al., 2017). In the United Kingdom, it was estimated that more than $£ 180$ million was spent on BPH treatments each year (Speakman et al., 2015). In 2000, it was estimated that the direct cost of medical services for BPH in the United Sates was about US\$1.1 billion (Wei et al., 2005). In Hong Kong, the per capita total expenditure on health in 2010/2011 was HK\$ 13302 (US\$ 1705) (Department of Health, 2017). Like many places around the world, the healthcare systems local and abroad face an increasing cost scrutiny. Cost-effectiveness assessment of treatments is gaining more importance to ensure patient satisfaction as well as the most efficient outcome in the environment of costcontainment.

In view of this, the objective of our study was to evaluate the long-term cost-effectiveness of oral, daily, singledose combination therapy dutasteride/tamsulosin (Duodart ${ }^{\circledR}$ ), compared with oral, daily tamsulosin 0.4 mg in Hong Kong (HK).

\section{MATERIALS AND METHODS}

\section{Overview of the Economic Model}

A Markov model was developed to project the overall costsavings of combination therapy in a hypothetical cohort of 10,000 patients treated in the public healthcare sector of HK. As such, ethical approval was not obtained since no patient was involved in this research. The natural history of $\mathrm{BPH}$ progression has been widely examined in literature (Barry, 1990; Wei et al., 2005). As such, we used this as

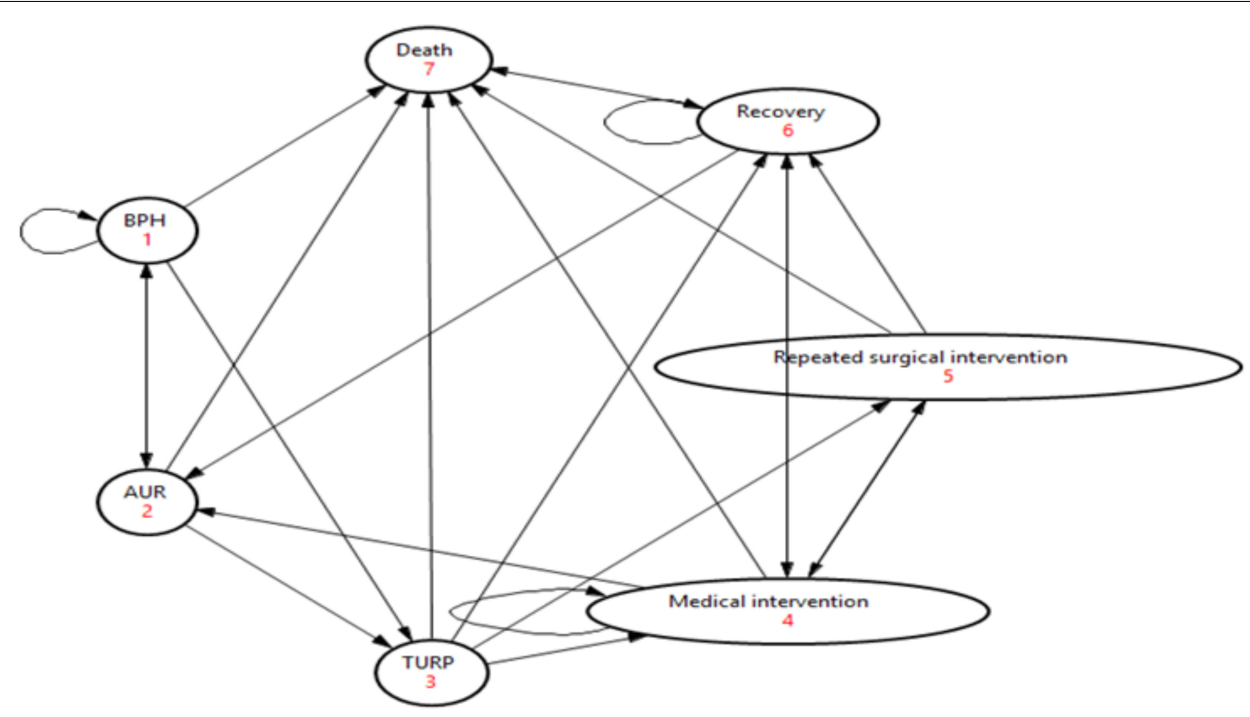

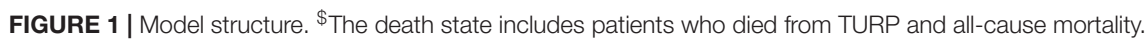


TABLE 1 | Model inputs.

\begin{tabular}{|c|c|c|c|}
\hline Parameters & Base-case & Range & Source \\
\hline \multicolumn{4}{|l|}{ Transition probability (monthly) } \\
\hline Remaining in $\mathrm{BPH}$ & 0.345 & $0.248-0.45$ & Ball et al., 1981 \\
\hline BPH patients who experience TURP & 0.021 & $0.0098-0.036$ & Ball et al., 1981 \\
\hline AUR patients going through TURP & 0.8 & $0.75-0.85$ & Prince of Wales Hospital^${ }^{\wedge}$ \\
\hline TURP patients' 30-day mortality & 0.0237 & $0.0179-0.0296$ & Madersbacher and Marberger, 1999 \\
\hline TURP patients who recover & 1 & $\mathrm{~N} / \mathrm{A}$ & Madersbacher and Marberger, 1999 \\
\hline TURP patients who require medical intervention & 0.059 & $\mathrm{~N} / \mathrm{A}$ & Prince of Wales Hospital $\%$ \\
\hline TURP patients who require repeated surgical intervention & 0.059 & $N / A$ & Prince of Wales Hospital $\%$ \\
\hline Patients with repeated TURP died from surgical procedure & 0 & N/A & Prince of Wales Hospital $\%$ \\
\hline Patients who remain at recovery state & 0.329 & $0.275-0.41$ & Prince of Wales Hospital \\
\hline Recovered patients who require medical intervention & 0.0467 & $0.0295-0.0744$ & Prince of Wales Hospital \\
\hline Patients on medication who require the second TURP & 0.036 & $0.0215-0.061$ & Prince of Wales Hospital \\
\hline Patients with repeated TURP requiring medication intervention & 0.0025 & $\mathrm{~N} / \mathrm{A}$ & Prince of Wales Hospital $\%$ \\
\hline Patients with repeated TURP who recover & 1 & N/A & Prince of Wales Hospital $\%$ \\
\hline Patients remain on medication & 0.393 & $0.378-0.438$ & Prince of Wales Hospital $\%$ \\
\hline TURP patients who fully recover & 0.998 & $0.978-1$ & Prince of Wales Hospital $\%$ \\
\hline Patients on medication who transition to AUR state & 0 & & Ball et al., 1981 \\
\hline BPH patients who experience AUR & 0.004 & $0.00064-0.0154$ & Ball et al., 1981 \\
\hline All-cause mortality & & & HK life table \\
\hline \multicolumn{4}{|l|}{ Efficacy (over 4 years) } \\
\hline $\begin{array}{l}\text { Efficacy of tamsulosin/dutasteride against AUR (vs. } \\
\text { monotherapy) }\end{array}$ & 0.676 & $0.527-0.778$ & Roehrborn et al., 2010 \\
\hline $\begin{array}{l}\text { Efficacy of tamsulosin/dutasteride against TURP (vs. } \\
\text { monotherapy) }\end{array}$ & 0.706 & $0.577-0.795$ & Roehrborn et al., 2010 \\
\hline \multicolumn{4}{|l|}{ Cost (per annum) (2018 US\$) ${ }^{@}$} \\
\hline tamsulosin/dutasteride & 464.97 & $348-581$ & Hong Kong public hospital formulary estimation \\
\hline Tamsulosin & 55.79 & $41.8-69.7$ & Hong Kong public hospital formulary estimation \\
\hline Managing a patient who is initially in $\mathrm{BPH}$ moderate state & 471 & $223-720$ & Prince of Wales Hospital $\%$ \\
\hline Managing a patient who experiences an episode of AUR & 1312 & $590-4,199$ & Prince of Wales Hospital $\%$ \\
\hline TURP procedure & 6334 & $4,549-8,119$ & Prince of Wales Hospital $\%$ \\
\hline Managing a patient who requires medical intervention & 371 & $123-620$ & Prince of Wales Hospital $\%$ \\
\hline \multicolumn{4}{|l|}{ Utility } \\
\hline $\mathrm{BPH}$ mild & 0.993 & $0.94-1$ & Baladi et al., 1996 \\
\hline BPH moderate & 0.903 & $0.86-0.95$ & Baladi et al., 1996 \\
\hline $\mathrm{BPH}$ severe & 0.79 & $0.75-0.83$ & Baladi et al., 1996 \\
\hline $\mathrm{BPH}$ (weighted) (used in the model) ${ }^{\$}$ & 0.876 & $0.83-0.92$ & Baladi et al., 1996 \\
\hline AUR & 0.25 & $0.24-0.26$ & Cher et al., 1997 \\
\hline TURP\# & 0.25 & $0.24-0.26$ & Cher et al., 1997 \\
\hline Medical intervention & 0.25 & $0.24-0.26$ & Assumption \\
\hline Recovery & 1.0 & $0.95-1$ & Assumption \\
\hline
\end{tabular}

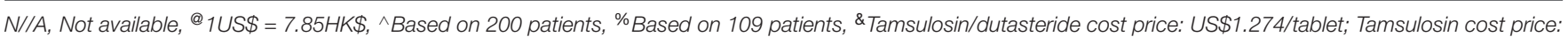

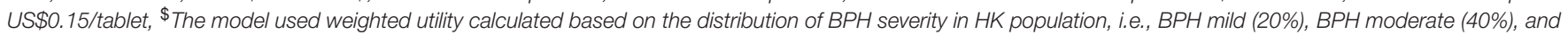
$\mathrm{BPH}$ severe (40\%), "The utility of a patient undergoing the second TURP is considered the same as that of the first TURP.

our base case to build our model. We subsequently had a discussion workshop to seek clinical experts' views on the disease progression pathway of $\mathrm{BPH}$ and to understand the use of different treatments in the local context. The workshop included review of international BPH management guidelines, exploration of consensus in the local clinical setting, as well as retrospective analysis of $\mathrm{BPH}$ outcome data from the territory. The model population is consistent with the entry criteria for the CombAT trial (Roehrborn et al., 2010), i.e., the hypothetical cohort of patients $\geq 50$ year of age with a $\mathrm{BPH}$ clinical diagnosis by medical history and physical examination. This age group was representative of $\mathrm{BPH}$ patient population in Asia and the most commonly studied age group in clinical trials.

The model was structured according to the HK-specific treatment practice, and is shown in Figure 1. In the beginning of the simulation, patients start from BPH state. As time progresses, patients might remain in $\mathrm{BPH}$ state, transition between the symptom states, experience AUR, receive a surgical intervention (TURP), or die due to natural causes. After experiencing an 
TABLE 2 | Model inputs and their distributions used in multivariate probabilistic sensitivity analysis.

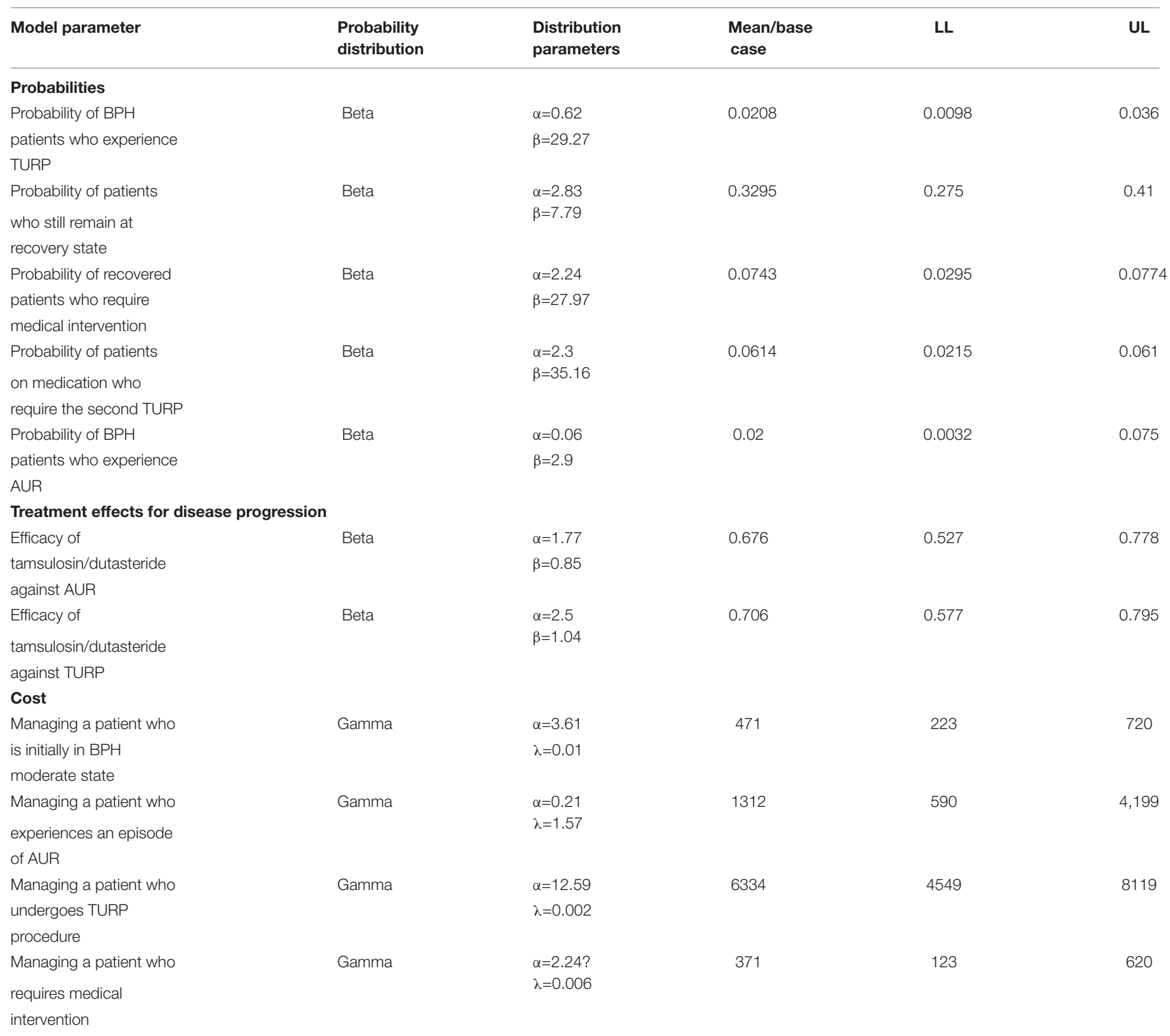

LL, Lower limit; UL, Upper limit.

AUR episode, the patient could transition back to $\mathrm{BPH}$ state. After TURP, a patient would receive medical intervention or have a repeated TURP procedure if the initial surgical procedure is not successful. Patients who have undergone TURP could die due to complications of the procedure, or they could die due to other causes. A patient in TURP, repeated TURP or medical intervention state could recover back to healthy state.

The analysis was performed from the healthcare payers' perspective, which only included direct costs. A cycle length of 1 year was adopted to capture the full effect of both intervention in terms of resource use and quality of life. In the base-case scenario, the model was simulated over 4 years, i.e., duration of the trial. To assess the sensitivity of the model results to the time horizon, the model was also run for 35 years, i.e., patients' life span. A discount rate of $3 \%$ was applied to both cost and outcome.

\section{Assumptions}

There are a few assumptions made in our model as described in the following:

(1) In reality, $\mathrm{BPH}$ state can be classified according to severity, i.e., mild, moderate, and severe states. However, due to the lack of granular data on symptom severity as measured by the International Prostate Symptom Score (IPSS) of BPH patients, we modeled patients into one $\mathrm{BPH}$ state; 
TABLE 3 | Base-case results.

\begin{tabular}{|c|c|c|c|}
\hline & $\begin{array}{c}\text { Combination } \\
\text { therapy (a) }\end{array}$ & $\begin{array}{l}\text { Monotherapy } \\
\text { (b) }\end{array}$ & $\begin{array}{c}\text { Difference } \\
\text { (a-b) }\end{array}$ \\
\hline \multicolumn{4}{|c|}{ Model time horizon: 4 years } \\
\hline \multicolumn{4}{|c|}{ Average cost per patient of 10,000 population (US\$ 2018) } \\
\hline Drug & 1,749 & 206 & 1,543 \\
\hline $\mathrm{BPH}$ & 1,935 & 1,642 & 293 \\
\hline AUR & 108 & 139 & -31 \\
\hline TURP & 1,090 & 2,119 & $-1,029$ \\
\hline Medical intervention & 6 & 8 & -2 \\
\hline \multicolumn{4}{|c|}{ Health outcomes per patient (by episode) } \\
\hline $\mathrm{BPH}$ & 3.43 & 3.3 & 0.15 \\
\hline AUR & 0.08 & 0.11 & -0.03 \\
\hline TURP & 0.22 & 0.29 & -0.07 \\
\hline Death & 0.056 & 0.0577 & -0.002 \\
\hline QALY & 3.00 & 2.93 & 0.07 \\
\hline \multicolumn{4}{|c|}{ Incremental cost-effectiveness ratio } \\
\hline Cost per QALY gained & 11,651 & & \\
\hline \multicolumn{4}{|c|}{ Model time horizon: Life-time (35 years) } \\
\hline \multicolumn{4}{|c|}{ Average cost per patient of 10,000 population (US\$ 2018) } \\
\hline Drug & 7,473 & 856 & 6,617 \\
\hline $\mathrm{BPH}$ & 5,224 & 4,475 & 749 \\
\hline AUR & 2,644 & 3,095 & -451 \\
\hline TURP & 6,124 & 11,594 & $-5,470$ \\
\hline Medical intervention & 212 & 249 & -37 \\
\hline \multicolumn{4}{|c|}{ Health outcomes per patient (by episode) } \\
\hline $\mathrm{BPH}$ & 11.09 & 9.5 & 1.59 \\
\hline AUR & 2.01 & 2.36 & -0.35 \\
\hline TURP & 2.25 & 2.62 & -0.37 \\
\hline Death & 0.9675 & 0.9784 & 0.0109 \\
\hline QALY & 10.29 & 9.87 & 0.42 \\
\hline \multicolumn{4}{|c|}{ Incremental cost-effectiveness ratio } \\
\hline Cost per QALY gained & 3,329 & & \\
\hline
\end{tabular}

(2) It is assumed that high proportion of acute urinary retention (AUR) patients $(80 \%)$ will receive transurethral resection of the prostate (TURP) procedure. This uncertainty will be addressed using 1-way and multivariate probabilistic sensitivity analyses;

(3) Patients who recover from the first TURP procedure are assumed to incur only one follow-up cost. Medical intervention means the use of pharmacological agents for follow-up management;

(4) Post-TURP death due to TURP-related complications is defined as death which occurs within 30 days after TURP procedure;

(5) The second TURP procedure will require different amount of resources compared to the first TURP; Based on local literature, post-TURP death due to second TUPR is assumed to be the same as that from first TURP;

(6) It is assumed that patients will receive at most 2 TURP procedures.

\section{Data Inputs}

As summarized in Table 1, model parameters including transition probabilities, efficacy, costs, and utilities were derived from HK-specific sources, literatures and assumptions based on clinical inputs.

The majority of the transition probabilities were derived from a cohort of $200 \mathrm{BPH}$ patients from Prince of Wales Hospital. Those patients comprised of roughly $10 \%$ of overall $\mathrm{BPH}$ patients in HK thus were considered representative of all BPH patients' profile in HK. The remaining probabilities were obtained from published literature where available. In addition to mortality due to TURP procedure, the model also allowed for patients to die from all-cause mortality which was obtained from HK life table. The efficacy of the different interventions were derived from a 4-years, multicentre, randomized, double-blind, parallelgroup CombaT clinical trial which included 4,844 men $\geq 50$ years of age with a clinical diagnosis of $\mathrm{BPH}$, International Prostate Symptom Score $\geq 12$ (Roehrborn et al., 2010). The acquisition cost price of combination therapy and tamsulosin were US\$1.274 and US\$0.15 per tablet respectively based on the acquisition cost of Prince of Wales Hospital, which is a public hospital. The costs are relatively similar across all public hospitals in Hong Kong, which account for the care of majority of patients with $\mathrm{BPH}$ in the territory. Similarly, the costs of management of $\mathrm{BPH}, \mathrm{AUR}$, TURP, and medical intervention were all solicited from Prince of Wales Hospital. Quality of life (utility) values associated with each health state were extrapolated from two other BPH cost-effectiveness studies (Baladi et al., 1996; Cher et al., 1997) as local utility study was not available. In the model, QALYs were calculated by applying the utility to the survival duration of a patient from his/her health state. There was significant reduction of utility for patients experiencing AUR and having TURP procedure compared with patients at $\mathrm{BPH}$ state.

\section{Assessment Methods of Outcomes}

Outcome measure is expressed as incremental cost per qualityadjusted life year (QALY) gained. As of now, there is no costeffectiveness threshold in Hong Kong, 3 times Hong Kong GDP per capita 2017 (US\$45,887) recommended by WHO-CHOICE (Evans et al., 2005) The CHOICE (CHOosing Interventions that are Cost-Effective) was used to determine if the new intervention is cost-effective compared to the existing one (Economic Analaysis Division Finance Secretary's Office, 2018).

\section{Deterministic One-Way Sensitivity Analysis (OWSA)}

To identify key model parameters, OWSA was conducted over the range of pre-defined values of each parameter's point estimate (i.e., 95\% confidence interval). Results were plotted in a tornado diagram according to the extent of the parameter's impact on the incremental cost-effectiveness ratio (ICER).

\section{Multivariate Probabilistic Sensitivity Analysis}

To assess the simultaneous influence of model parameters on the ICER, a multivariate probabilistic sensitivity analysis was performed using second-order Monte Carlo simulation (computational algorithm based on repeated random sampling 


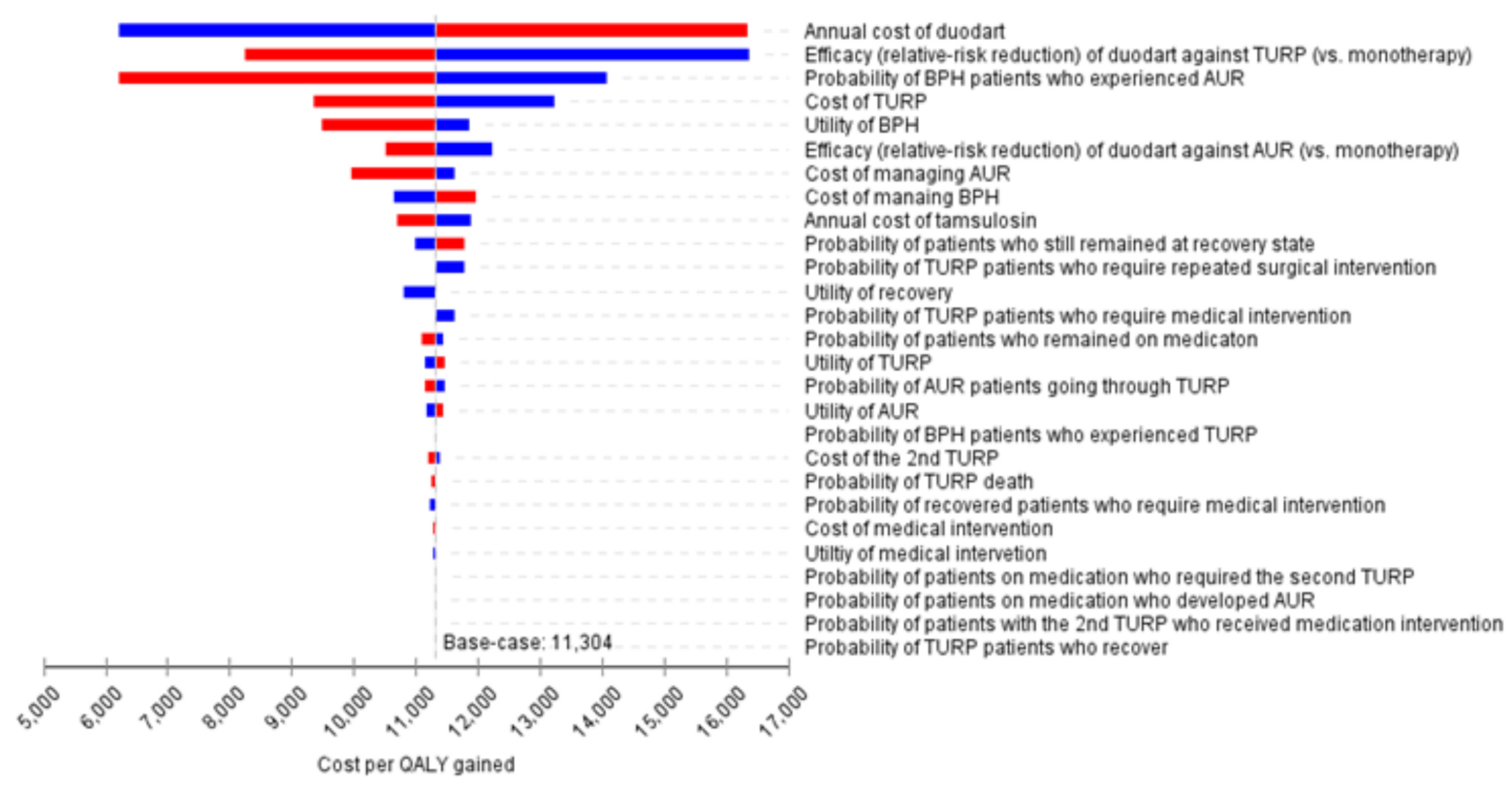

Efficacy (relative-risk reduction) of duodart against TURP (vs. monotherapy) Probability of BPH patients who experienced AUR Cost of TURP tility of $\mathrm{BPH}$

Efficacy (relative-risk reduction) of duodart against AUR (vs. monotherapy)

Pre remained at recover state

Probability of TURP patients who require repeated surgical intervention

Utility of TURP

Utobability of AUR patients going through TURP

Utility of AUR

Probability of BPH patients w

Probabily of recovered patients who require medical intervention

second TURP

Probability of patients on medication who developed AUR

who received medication intervention

FIGURE 2 | Tornado diagram for one-way sensitivity analysis. "The results were based on 4-year projection.

of the probability distributions for each model parameter). A total of 10,000 Monte-Carlo iterations were simulated, each generating a model-estimated value for the cost and QALY. The cost-effectiveness plane was produced depicting the scatterplot of the 10,000 simulated sets of cost and QALY estimates. In addition, a cost-effectiveness acceptability curve (CEAC) was generated to display the probability of cost-effectiveness of both interventions at each willingnessto-pay threshold. Table 2 presents the categories of model parameters, probability distributions as well as the upper and lower values used in the multivariate probabilistic sensitivity analysis.

The model was implemented in TreeAge pro 2017 (TreeAge Software, Williamstown, MA, United States). A microsimulation of 10,000 patients was executed to enable the tracking of the number of clinical events.

\section{RESULTS}

\section{Base-Case Analysis}

As shown in Table 3, the Markov microsimulation based on 10,000 iterations showed that, over 4 years, compared to tamsulosin, combination therapy could prevent 0.03 AURs ( $\sim 27 \%$ reduction) per patient, 0.07 TURPs $(\sim 32 \%$ reduction) per patient, and 0.002 deaths $(\sim 3.5 \%$ reduction $)$ over 4 years. Compared to tamsulosin, combination therapy can lead to cost reduction of US $\$ 31$, US $\$ 1,029$, and US $\$ 2$ per patient due to reduced episodes of AUR, TURP and need of medical intervention, respectively and additionally a delay in patient's progression to AUR and TURP. However, those benefits came with an incremental drug cost of US\$1,543 per patient over 4-year period. The increased number of $\mathrm{BPH}$ (3.43) for combination therapy compared to that (3.3) of monotherapy was mainly due to the delayed progression of $\mathrm{BPH}$ patients to AUR and TURP health states. In addition, the analysis showed that a patient on combination therapy would experience more QALYs (3 vs. 2.93 QALYs) than those on monotherapy. As a result, replacing monotherapy by combination therapy was expected to lead to US\$11,651 per QALY gained.

As the modeling time horizon was extended to life-time, compared to monotherapy, more health benefits were projected for patients receiving combination therapy, i.e., a reduction of 0.35 AURs per patient, 0.37 TURPs per patient and 0.0109 deaths, which translated into 0.42 QALY gained per patient. In addition, using combination therapy at an incremental drug cost of US $\$ 6,617$ compared to tamsulosin could potentially save US $\$ 451$ per patient in managing AUR and US\$5,470 per patient in TURP procedure. Similarly, as the combination therapy reduced risks of a patient progressing to AUR and TURP health states, therefore, an incremental cost of US\$749 in managing BPH was incurred.

\section{Way Sensitivity Analysis}

The OWSA showed that the top five most influential parameters on ICER were annual cost of tamsulosin/dutasteride, efficacy of tamsulosin/dutasteride against TURP, and probability of $\mathrm{BPH}$ patients who experienced AUR, cost of providing TURP procedure and utility of $\mathrm{BPH}$. The remaining parameters had only a moderate influence on ICER estimates causing ICER to vary by no more than US\$2,000. On the whole, all the ICERs were found to be between US\$6,000 and US\$17,000 per QALY when parameters were varied over their uncertainty range (Figure 2). 


\section{A Projection over 4 years}

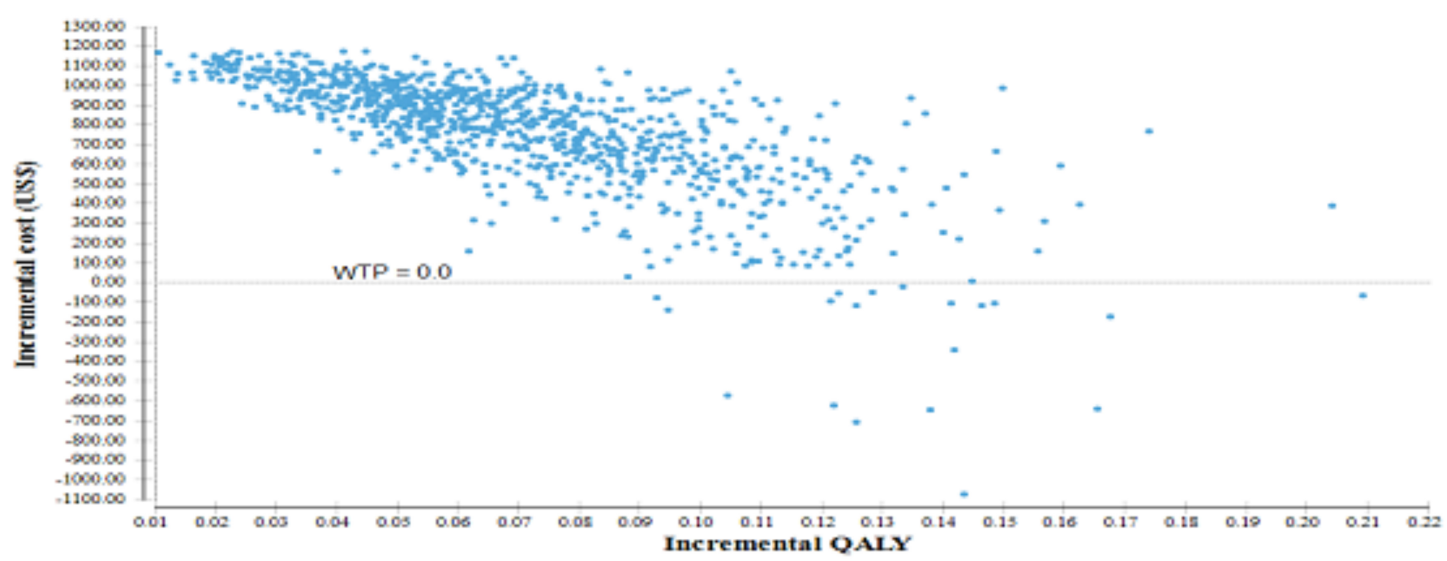

B Projection over life-time

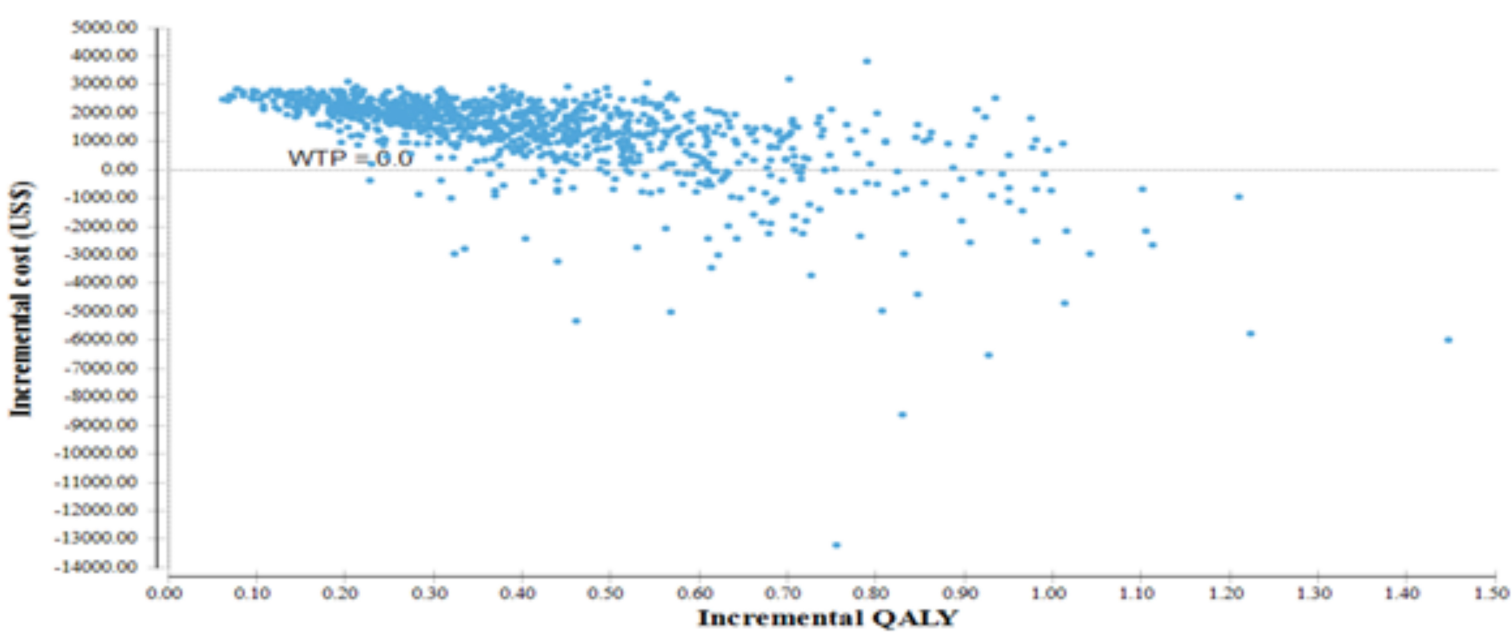

FIGURE 3 | Cost-effectiveness scatterplot. (A) Cost effectiveness projection over 4 years. (B) Cost effectiveness projection over life-time.

\section{Multivariate Probabilistic Sensitivity Analysis}

The average incremental costs and QALYs over the 10,000 Monte Carlo simulations from the probabilistic sensitivity analysis were essentially similar compared to the deterministic basecase results. As shown in Figure $\mathbf{3 A}$, over 4-year projection period, the scatterplots of incremental costs and QALYs for combination therapy vs. monotherapy showed that over $98 \%$ of the 10,000 Monte Carlo iterations fell within the north-east quadrant, suggesting that combination therapy produced higher QALYs at a higher cost, even with uncertainty in all parameters. In Figure 3B, over life-time horizon, there was a trend suggesting that combination therapy was cost-saving, i.e., less costly and more effective, among $12.8 \%$ of all 10,000 simulations while the remaining simulated results fell within the north-east quadrant.

As shown in Figure 4, the CEAC showed that, over 4year time horizon, it demonstrated that the probability of combination therapy being cost-effective was greater than $95 \%$ if willingness to pay (WTP) threshold is above US\$40,000. Besides, the combination therapy had a probability of $97 \%$ of being costeffective over monotherapy at one time HK GDP per capita of US $\$ 45,887$. Over life-time projection, combination therapy would have a probability of cost-effectiveness of more than 95\% if WTP threshold is above US $\$ 20,000$. It will achieve a probability of $99 \%$ being cost-effective at one time HK GDP per capita.

\section{DISCUSSION}

Hong Kong's healthcare system comprises of the public and private sector, with the former being the cornerstone of the healthcare system, acting as a safety net for the population. The public system currently serves $90 \%$ of all inpatient needs and $\sim 30 \%$ of outpatient services (Kong et al., 2015). It has a very comprehensive range of services which are charged at very low rates, representing approximately 95\% subsidy from the actual cost. Similar to many countries around the world, Hong Kong 


\section{A}

Projection over 4 years

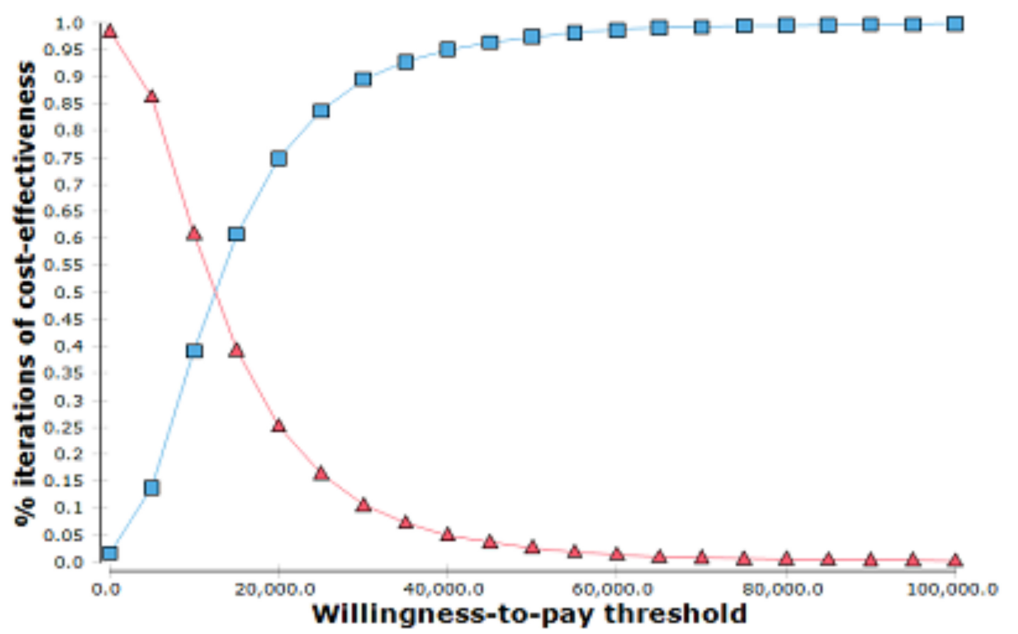

Combination therapy

$\Delta$ Monotherapy

B

Projection over life-time

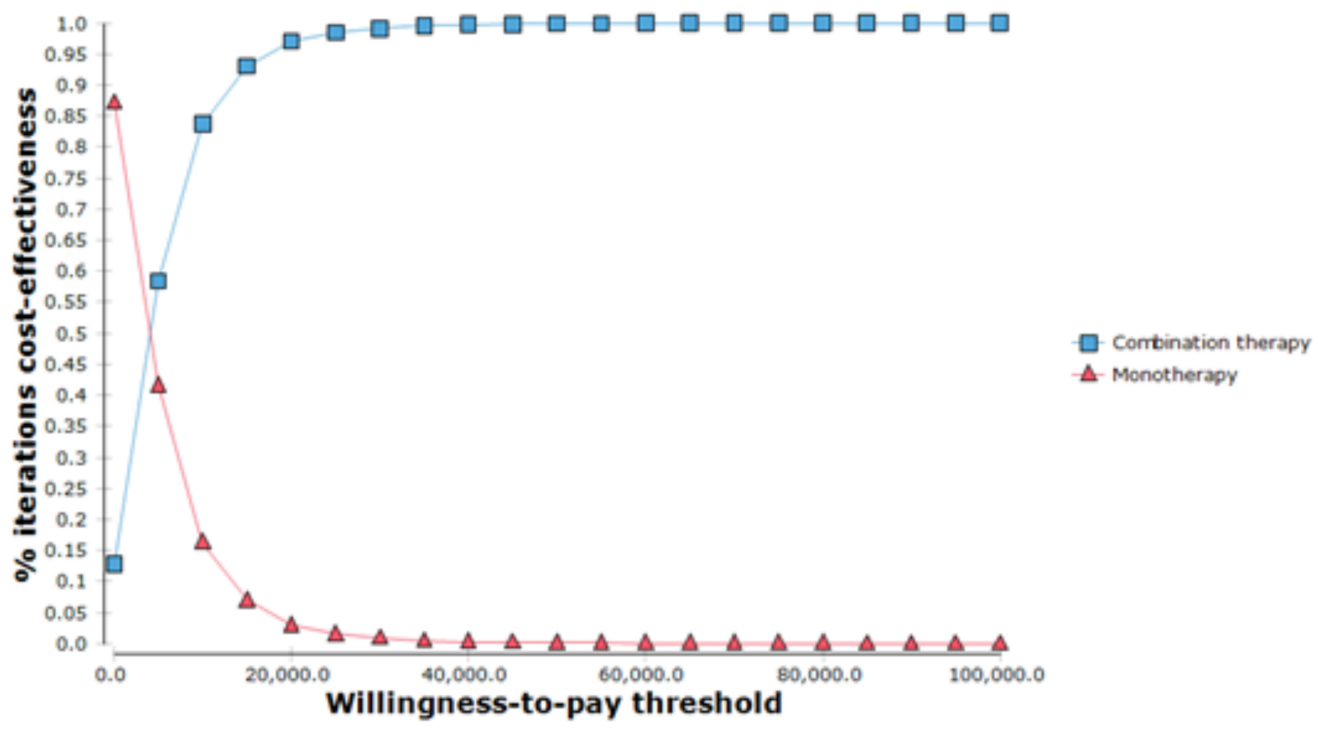

FIGURE 4 | Cost effectiveness acceptability curve. (A) Projection over a 4 year period. (B) Projection over lifetime period.

is currently facing serious challenges due to its rapidly aging population (Kong et al., 2015; Lee and Mak, 2016; Ang et al., 2017). It is estimated that the total population aged $>60$ years in Hong Kong will rise from $12.5 \%$ currently to $25 \%$ in 2030 , which will thus result in an increase in BPH prevalence. 5ARIs and alpha blockers, used either as monotherapy or combination therapy, are common modalities for BPH/LUTS treatment. While the results of the CombAT trial proved the clinical benefits of dutasteride/tamsulosin combination in symptom relief and reducing clinical progression of BPH (Roehrborn et al., 2010), our model based on current drug price showed that patients who were treated with combination therapy experienced more
QALYs but incurred higher costs than patients on background medications, leading to an ICER of US\$11,651 and US\$3,329 per QALY over 4-year and life-time horizon. Deterministic OWSA indicated that the results were most sensitive to the acquisition cost of combination therapy and drug efficacy.

It is worth noting that based on hospital data $80 \%$ of all patients with AUR would eventually go through the most common surgical procedure for BPH treatment, i.e., TURP. This is reflective of the reality as a small number of patients would not be fit for surgery due to various medical reasons, and besides TURP there are other surgical options for the management of TURP, e.g., laser prostatectomy. However, in the setting of 
the local health care system, TURP is still the majority. Such assumption for the construction of the Markov model would give a good estimation of the results.

Despite the differences in epidemiology and costs, our findings were consistent with the other published cost-effectiveness analyses which also showed that combination therapy led to the potential incremental cost and benefit of QALYs gained, leading to cost-effectiveness of the new intervention. For example, a study in Canady by Ismaila et al. (2013) showed that, over a lifetime, the combination therapy led to an ICER of US\$20,224 per QALY gained over 10-year time horizon and was considered cost-effective for $99.6 \%$ of probability at a willingness-to-pay threshold of CAN\$50,000 (US\$39,752) per QALY. In the United Kingdom, Walker et al. (2013) constructed a Markov state transition model measured by QALYs for patients aged $\geq 50$ years with $\mathrm{BPH}$ and moderate to severe symptoms. While cumulative discounted costs per patient were higher with combination therapy than with tamsulosin alone, QALYs were also higher. Their probabilistic sensitivity analysis revealed that the probability of combination therapy being cost-effective lied in the range of $78-88 \%$. In a Norwegian economic model, Bjerklund Johansen et al. (2012) used a semi-Markov model to project costs and utility outcomes over two time horizons, namely 4 years and lifetime. ICERs of combination were US\$12,374 and US\$6,871 over 4 years and lifetime, respectively. Another Japanese study on the pharmacoeconomic evaluation of combination therapy suggested that such treatment with alpha blocker and dutasteride in $\mathrm{BPH}$ patients would be more costeffective than alpha blockers alone in patients with moderate to severe symptoms (Takayama et al., 2012). The ICERs for combination therapy versus monotherapy calculated at 4 years and 10 years were US\$47,581 and US\$55,532/QALY gained, respectively, both below the acceptable ICER threshold in Japan.

The present study has several limitations. Firstly, in our Markov model, due to data availability, we did not separate the state of symptomatic BPH into patients with mild, moderate and severe symptoms according to IPSS score. Instead, these 3 groups of patients were analyzed as a single group. Such categorization might have made the prediction of AUR probability less precise. As shown in OWSA, the utility of BPH has substantial impact on ICER. Future study on classification of BPH severity was thus warranted. Secondly, we didn't model the occurrence of adverse events because, as reported in the ComBAT trial, the incidences of adverse events were very similar between combination therapy and monotherapy. In a 2015 study, complete absence of ejaculation was experienced by $23 \%$ of patients on combination

\section{REFERENCES}

Ang, B. H., Chen, W. S., and Lee, S. W. H. (2017). Global burden of road traffic accidents in older adults: a systematic review and meta-regression analysis. Arch. Gerontol. Geriatr. 72, 32-38. doi: 10.1016/j.archger.2017.05.004

Baladi, J.-F., Menon, D., and Otten, N. (1996). An economic evaluation of finasteride for treatment of benign prostatic hyperplasia. Pharmacoeconomics 9, 443-454. therapy, and 15\% on tamsulosin alone (Stojanoviæ et al., 2015). In the same study, it was found that erection improved in both groups of the patients. Overall, the difference in adverse events between the 2 groups is very small and is expected to have minimal impact on the cost-effectiveness results. Thirdly, we didn't provide analysis from societal perspective which includes indirect costs associated with $\mathrm{BPH}$ patients. Indirect costs associated with $\mathrm{BPH}$ would include cost from complications of BPH e.g., urinary tract infection, bladder stones, and hematuria (Leong et al., 2014; Lee et al., 2015). It also would include the loss of productivity of patients and caregivers. The inclusion of those costs is expected to make the cost-effective results even more favorable. Finally, our model were based upon data from literature which dates back to 1990s. While this may not be truly representative of the current situation, we had to rely on such classical studies for the input of the model. Nevertheless, we had also sought experts input to ensure that the model was representative of the current situation in Hong Kong to ensure its applicability.

In conclusion, our study shows that combination therapy as compared with monotherapy is cost-effective due to substantial reduction in the number of AUR and TURP and the associated direct cost in $\mathrm{BPH}$ patients in Hong Kong.

\section{DATA AVAILABILITY}

Data sharing is not applicable to this article as no datasets were generated or analyzed during the current study.

\section{AUTHOR CONTRIBUTIONS}

DW played a principal role in the study concept and design, performed the statistical analysis, interpreted the results, and wrote the draft. CY designed the study, provided statistical support, reviewed, and edited the manuscript. C-FN, KL, SL, and NC contributed to data acquisition or interpretation of data. Y-SC provided inputs in reviewing the methodology part and assisted with model calibration using TreeAge software. KL obtained the funding. All authors approved the final manuscript.

\section{FUNDING}

The publication is based on a research funded by GlaxoSmithKline Hong Kong.

Ball, A. J., Feneley, R. C. L., and Abrams, P. H. (1981). The natural history of untreated “prostatism”. Br. J. Urol. 53, 613-616. doi: 10.1111/j.1464-410X.1981. tb03273.x

Barry, M. (1990). Epidemiology and natural history of benign prostatic hyperplasia. Urol. Clin. North Am. 17, 495-507.

Bjerklund Johansen, T. E., Baker, T. M., and Black, L. K. (2012). Costeffectiveness of combination therapy for treatment of benign prostatic hyperplasia: a model based on the findings of the combination of Avodart 
and Tamsulosin trial. BJU Int. 109, 731-738. doi: 10.1111/j.1464-410X.2011. 10511.x

Cher, D. J., Miyamoto, J., and Lenert, L. A. (1997). Incorporating risk attitude into Markov-process decision models: importance for individual decision making. Med. Decis. Making 17, 340-350.

Department of Health (2017). Health Facts of Hong Kong 2017 Edition. Available at: http://www.dh.gov.hk/english/statistics/statistics_hs/files/Health_Statistics_ pamphlet_E.pdf [accessed February 4, 2018].

Economic Analaysis Division Finance Secretary's Office (2018). 2017 Economic Background and 2018 Prospects - Statistical Appendix. Hong Kong: Government of the Hong Kong Special Administrative Region.

Evans, D. B., Edejer, T. T.-T., Adam, T., and Lim, S. S. (2005). Methods to assess the costs and health effects of interventions for improving health in developing countries. BMJ 331, 1137-1140.

Ismaila, A., Walker, A., Sayani, A., Laroche, B., Nickel, J. C., Posnett, J., et al. (2013). Cost-effectiveness of dutasteride-tamsulosin combination therapy for the treatment of symptomatic benign prostatic hyperplasia: a Canadian model based on the CombAT trial. Can. Urol. Assoc. J. 7, E393-E401. doi: 10.5489/cuaj. 12131

Kaplan, A. L., Agarwal, N., Setlur, N. P., Tan, H. J., Niedzwiecki, D., Mclaughlin, N., et al. (2015). Measuring the cost of care in benign prostatic hyperplasia using time-driven activity-based costing (TDABC). Healthcare 3, 43-48. doi: 10.1016/ j.hjdsi.2014.09.007

Kong, X., Yang, Y., Gao, J., Guan, J., Liu, Y., Wang, R., et al. (2015). Overview of the health care system in Hong Kong and its referential significance to mainland China. J. Chin. Med. Assoc. 78, 569-573. doi: 10.1016/j.jcma.2015.02.006

Lee, S. W.-H., Chaiyakunapruk, N., Chong, H.-Y., and Liong, M.-L. (2015). Comparative effectiveness and safety of various treatment procedures for lower pole renal calculi: a systematic review and network meta-analysis. BJU Int. 116, 252-264. doi: 10.1111/bju.12983

Lee, S. W. H., Chan, E. M. C., and Lai, Y. K. (2017). The global burden of lower urinary tract symptoms suggestive of benign prostatic hyperplasia: a systematic review and meta-analysis. Sci. Rep. 7:7984. doi: 10.1038/s41598-017-06628-8

Lee, S. W. H., Liong, M. L., Yuen, K. H., Leong, W. S., Cheah, P. Y., Khan, N. A., et al. (2008). Adverse impact of sexual dysfunction in chronic prostatitis/chronic pelvic pain syndrome. Urology 71, 79-84. doi: 10.1016/j. urology.2007.08.043

Lee, S. W. H., Liong, M. L., Yuen, K. H., Liong, Y. V., and Krieger, J. N. (2007). Chronic prostatitis/chronic pelvic pain syndrome: role of alpha blocker therapy. Urol. Int. 78, 97-105.

Lee, S. W. H., and Mak, V. S. L. (2016). Changing demographics in Asia: a case for enhanced pharmacy services to be provided to nursing homes. J. Pharm. Pract. Res. 46, 152-155.

Leong, W. S., Liong, M. L., Liong, Y. V., Wu, D. B.-C., and Lee, S. W. H. (2014). Does simultaneous inversion during extracorporeal shock wave lithotripsy improve stone clearance: a long-term, prospective, single-blind, randomized controlled study. Urology 83, 40-44. doi: 10.1016/j.urology.2013.08.004

McVary, K. T., Roehrborn, C. G., Avins, A. L., Barry, M. J., Bruskewitz, R. C., Donnell, R. F., et al. (2011). Update on AUA guideline on the management of benign prostatic hyperplasia. J. Urol. 185, 1793-1803. doi: 10.1016/j.juro.2011. 01.074

Madersbacher, S., and Marberger, M. (1999). Is transurethral resection of the prostate still justified? BJU Int. 83, 227-237.
Oelke, M., Bachmann, A., Descazeaud, A., Emberton, M., Gravas, S., Michel, M. C., et al. (2013). EAU guidelines on the treatment and follow-up of non-neurogenic male lower urinary tract symptoms including benign prostatic obstruction. Eur. Urol. 64, 118-140. doi: 10.1016/j.eururo.2013. 03.004

Roehrborn, C. G., Barkin, J., Siami, P., Tubaro, A., Wilson, T. H., Morrill, B. B., et al. (2011). Clinical outcomes after combined therapy with dutasteride plus tamsulosin or either monotherapy in men with benign prostatic hyperplasia (BPH) by baseline characteristics: 4-year results from the randomized, doubleblind Combination of Avodart and Tamsulosin (CombAT) trial. BJU Int. 107, 946-954. doi: 10.1111/j.1464-410X.2011.10124.x

Roehrborn, C. G., Siami, P., Barkin, J., Damião, R., Major-Walker, K., Nandy, I., et al. (2010). The effects of combination therapy with Dutasteride and Tamsulosin on clinical outcomes in men with symptomatic benign prostatic hyperplasia: 4-year results from the CombAT Study. Eur. Urol. 57, 123-131. doi: 10.1016/j.eururo.2009.09.035

Rosen, R., Altwein, J., Boyle, P., Kirby, R. S., Lukacs, B., Meuleman, E., et al. (2003). Lower urinary tract symptoms and male sexual dysfunction: the multinational survey of the aging male (MSAM-7). Eur. Urol. 44, 637-649.

Speakman, M., Kirby, R., Doyle, S., and Ioannou, C. (2015). Burden of male lower urinary tract symptoms (LUTS) suggestive of benign prostatic hyperplasia (BPH)-focus on the UK. BJU Int. 115, 508-519. doi: 10.1111/bju. 12745

Stojanović, N. A., Ignjatović, I., Đenić, N., and Bogdanović, D. (2015). Adverse effects of pharmacological therapy of benign prostatic hyperplasia on sexual function in men. Srp. Arh. Celok. Lek. 143, 284-289.

Takayama, T., Arakawa, I., Kakihara, H., Tachibana, K., and Ozono, S. (2012). Pharmacoeconomic evaluation of combination therapy with dutasteride and $\alpha 1$ blocker for treatment of benign prostatic hyperplasia in Japan. Hinyokika Kiyo 58, 61-69.

Walker, A., Doyle, S., Posnett, J., and Hunjan, M. (2013). Cost-effectiveness of single-dose tamsulosin and dutasteride combination therapy compared with tamsulosin monotherapy in patients with benign prostatic hyperplasia in the UK. BJU Int. 112, 638-646. doi: 10.1111/j.1464-410X.2012.11659.x

Wei, J. T., Calhoun, E., and Jacobsen, S. J. (2005). Urologic diseases in America project: benign prostatic hyperplasia. J. Urol. 173, 1256-1261.

Yee, C.-H., Li, J. K., Lam, H.-C., Chan, E. S., Hou, S. S.-M., and Ng, C.-F. (2014). The prevalence of lower urinary tract symptoms in a Chinese population, and the correlation with uroflowmetry and disease perception. Int. Urol. Nephrol. 46, 703-710. doi: 10.1007/s11255-013-0586-9

Conflict of Interest Statement: The authors declare that the research was conducted in the absence of any commercial or financial relationships that could be construed as a potential conflict of interest.

Copyright (c) 2018 Wu, Yee, Ng, Lee, Chaiyakunapruk, Chang and Lee. This is an open-access article distributed under the terms of the Creative Commons Attribution License (CC BY). The use, distribution or reproduction in other forums is permitted, provided the original author(s) and the copyright owner(s) are credited and that the original publication in this journal is cited, in accordance with accepted academic practice. No use, distribution or reproduction is permitted which does not comply with these terms. 